\title{
DST and DCT-based Depth of Interaction (DOI) Determining Techniques for LSO and LuYAP Scintillation Detectors in PET
}

\author{
H. Saleh \\ NCRRT, EAEA \\ Nasr City, Cairo \\ Egypt
}

\author{
A. Yahya \\ Electrical Engineering \\ Department, Al Azhar, \\ University, Nasr City, \\ Cairo, Egypt
}

\author{
M. Ashour \\ NCRRT, EAEA \\ Nasr City, Cairo Egypt
}

\author{
M. Sayed \\ NCRRT, EAEA \\ Nasr City, Cairo \\ Egypt
}

\begin{abstract}
The absorption of radiation in multicrystal PET detectors results in different decay-time pulses. The differences between these pulse decay time constants are used to discriminate between different scintillator materials. In this paper, pulse shape discrimination (PSD) techniques based on the discrete sine transform (DST) and discrete cosine transform (DCT) are proposed. These transformations are used to extract frequencydomain features for discrimination. Comparison of the proposed PSD techniques was performed on digitized pulses from the LSO/LuYAP phoswich detector. The comparison between different DST- and DCT-types turned out that DST-type 3 gives the best PSD efficiency of $98.79 \%$, while DCT-type 2 gives $98.12 \%$.
\end{abstract}

\section{General Terms}

Positron Emission Topography, algorithms.

\section{Keywords}

Pulse shape discrimination, DOI, DST, DCT

\section{INTRODUCTION}

Depth of Interaction (DOI) techniques allow discrimination between signals, which come from different layers of detectors. Different DOI techniques are used for crystal identification of phoswich detectors. This detector is a stack of two different scintillation materials optically coupled to a single photomultiplier tube (PMT) where each scintillator mainly responds to one radiation type.

Recent advancements of digital electronic architectures present high flexibility and reliability over analog technique ones [1]. This merit enables the development of new crystal identification algorithms for phoswich detectors. Such as in Pulse Shape Discrimination (PSD) techniques for neutron and gamma ray separation [2, 3], the incoming pulses from the detector are directly digitized and can be decomposed into individual pulses waveforms. As a result it can be easily getting the integration of two parts of the pulse from the digital waveforms [4, 5]. While in analog technique two separate charge-sensitive ADCs are required. One $\mathrm{ADC}$ is used to integrate the beginning of the pulse rise time while the second $\mathrm{ADC}$ is for integrating the tail part [6].
Different techniques are used to discriminate between the two different decay pulses such as constant fraction discrimination, in this technique the time between the time zero of pulses and time at which pulses amplitude crosses a fixed fraction of its maximum. When time are measured between upper and lower fraction, another technique called rise time discrimination was defined, while the normalized pulse amplitude at a fixed time after the beginning of pulse are used in the constant time discrimination technique [7]. In other technique, a parameter, which is proportional to the difference between the summation of the maximum energy of the pulse and the summation of the rest energy, is used to discriminate between two different decay pulses [8]. The Digital positron emission tomography (PET) techniques present high merits over analog ones [9-11]. These techniques allow the progress of different algorithms like wavelet and FFT based algorithms in [12-14] which will be compared to our proposed algorithms. Furthermore a method for identifying scintillating crystals in phoswich detectors based on cross-correlation has been investigated in [15].

PET spatial resolution is limited by a critical problem which known as parallax error. This error is caused by particles entering the detector with non-perpendicular angle of incidence. On the other hand, the problem is eliminated when the depth of interaction (DOI) is known [16]. There are many methods to obtain the depth of interaction information such as measuring the light spread on the detector $[17,18]$ or the ratio of light output on opposite ends of the scintillator crystal $[19,20]$.

This paper proposed two PSD techniques based on Discrete Sine Transform (DST) and Discrete Cosine Transform (DCT). In the following section, the pulse recording procedure is described as well as the DST and DCT are discussed. In section III, the two proposed DCT and DST-based algorithms are presented. The proposed techniques are compared and their results are discussed in section IV. Finally, section V gives the conclusions.

\section{PROCEDURE}

In this research work pulses are recorded from LSO/LuYAP detector, these pulses were digitized with a $25 \mathrm{~ns}$ sampling period while the trace length of $400 \mathrm{~ns}$ was accommodate for pulses. The sampled pulses were transferred to the host computer. These pulses were recorded by means of an acquisition board described in [1]. A set of 10000 pulses was recorded for two crystals (LSO, LuYAP). These pulses were amplified using PMT then filtered because the performance of the different PSD methods depends on the signal to noise ratio. 
Finally, the sampling frequency is $40 \mathrm{MHz}$, thus covering a time window of $400 \mathrm{~ns}$ where each pulse is represented by 16 samples. Fig. 1 shows averaged LSO and LuYAP pulses indicating that the LuYAP pulses have a significantly slow decay than the LSO pulses.

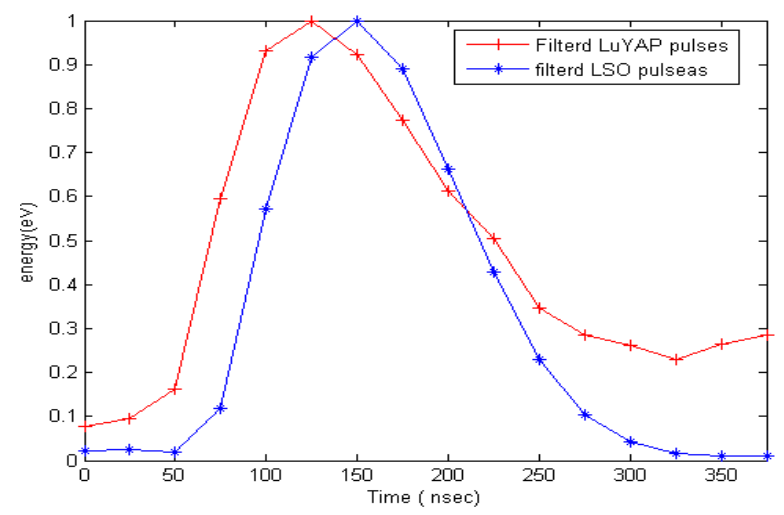

Figure 1: Normalized Filtered $3 \mathrm{MHz}$ and sampled pulses at $40 \mathrm{MHz}$ from LSO, LuYAP each averaged over all recorded events

\subsection{DST}

The DST is similar to the discrete Fourier transform (DFT), but using a purely real matrix. It is equivalent to the imaginary parts of a DFT of roughly twice the length, operating on real data with odd symmetry $[21,22]$. The $\mathrm{N}$ real numbers $\mathrm{X}_{0} \ldots \mathrm{X}_{\mathrm{N}-1}$ are transformed into the $\mathrm{N}$ real numbers $\mathrm{Y}_{0} \ldots \mathrm{Y}_{\mathrm{N}-1}$ according to one of the formulas:

DST-I

$$
Y_{k}=\sum_{n=0}^{N-1} x_{n} \sin \left[\frac{\pi}{N+1}(n+1)(k+1)\right]
$$

\section{DST-II}

$$
Y_{k}=\sum_{n=0}^{N-1} x_{n} \sin \left[\frac{\pi}{N}(n+1)(k+1)\right]
$$

\section{DST-III}

$$
Y_{k}=\frac{(-1)^{k}}{2} x_{N-1}+\sum_{n=1}^{N-2} x_{n} \sin \left[\frac{\pi}{N}(n+1)\left(k+\frac{1}{2}\right)\right]
$$

\section{DST-IV}

$$
Y_{k}=\sum_{n=0}^{N-1} x_{n} \sin \left[\frac{\pi}{N}\left(n+\frac{1}{2}\right)\left(k+\frac{1}{2}\right)\right]
$$

\subsection{DCT}

DCT is similar to the only real numbers discrete Fourier transform (DFT), by using even symmetry DCTs are equivalent to DFTs of roughly twice the length, operating on real data [23, 24]. The complexity of the calculating is the main parameter to choose the type of transform. There are several types of the DCT with slightly modified definitions. The $\mathrm{N}$ real numbers $\mathrm{X}_{0} \ldots \mathrm{X}_{\mathrm{N}-1}$ are transformed into the $\mathrm{N}$ real numbers $\mathrm{Y}_{0} \ldots \mathrm{Y}_{\mathrm{N}-1}$ according to one of the formulas:

\section{DCT-I}

$$
Y_{k}=\frac{1}{2}\left[x_{0}(-1)^{k} x_{N-1}\right]+\sum_{n=1}^{N-2} x_{n} \cos \left[\frac{\pi}{N-1} n k\right]
$$

DCT-II

$$
Y_{k}=\sum_{n=0}^{N-1} x_{n} \cos \left[\frac{\pi}{N}\left(n+\frac{1}{2}\right) k\right]
$$

\section{DCT-III}

\section{DCT-IV}

$$
Y_{k}=\frac{1}{2} x_{0}+\sum_{n=1}^{N-1} x_{n} \cos \left[\frac{\pi}{N}\left(k+\frac{1}{2}\right) n\right]
$$

$$
Y_{k}=\sum_{n=1}^{N-1} x_{n} \cos \left[\frac{\pi}{N}\left(k+\frac{1}{2}\right)\left(n+\frac{1}{2}\right)\right]
$$

\section{DST and DCT-based techniques}

In this paper, an efficient PSD technique, based on the frequency spectrum analysis, is proposed. The proposed technique uses the frequency domain, instead of time domain discriminations, in order to differentiate between the frequency components of the two different decay pulses. The transform type is studied under the many parameters. These parameters are the performance, and execution time, which indicates the efficiency of the calculating. By analyzing the different DCT and DST types at different level, the best one can be deduced to be used for a deduced separation of LSO from the LuYAP materials by notice the histogram of transformed pulses and find the best threshold value. Two methods are tested statistical equation method and tuned method.

\section{3-1 Statistical equation method:}

The mean $\left(\mu \operatorname{LSO}\left(\mu_{1}\right), \mu \operatorname{LuYAP}\left(\mu_{2}\right)\right)$ and the standard deviation $\left(\sigma \operatorname{LSO}\left(\sigma_{1}\right), \sigma \operatorname{LuYAP}\left(\sigma_{2}\right)\right)$ for each DST/DCT type at each level are calculated. There is a different threshold value at each level for each type which can be given by the following empirical equation:

$$
\begin{aligned}
& \text { Threshold }=\frac{c_{1} \mu_{1} \sigma_{2+} c_{2} \mu_{2} \sigma_{1}}{c_{2} \sigma_{1+} c_{1} \sigma_{2}} \\
& \text { Where } \begin{aligned}
c_{2}=\frac{2}{1+\sqrt{\frac{\sigma_{1}}{\sigma_{2}}}} \\
c_{1}=2-c_{2}
\end{aligned}
\end{aligned}
$$

The percentage is calculated using equation (12)

$$
\text { percentage }=\frac{N_{L S O}}{N_{A l l-L S O}}+\frac{N_{L u Y A P}}{N_{A l l-L u}} X \% 100
$$




\section{3-2 Tuned method:}

In this method, the threshold is chosen by scanning the intersection area of the two histograms of each output by small steps, and then the correctness percentage at each step is calculated to select the best.

\section{RESULTS AND DISCUSSIONS}

Figures 2, and 3 show the correctness percentages of Different types of DST and DCT-based techniques, respectively, applied over 10000 pulses for each transform type using MATLAB.

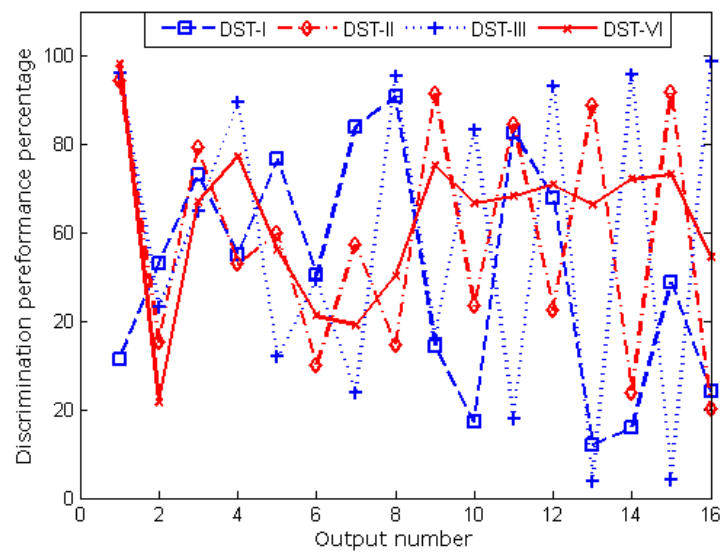

Figure 2 percentage efficiencies of the 4 DST types

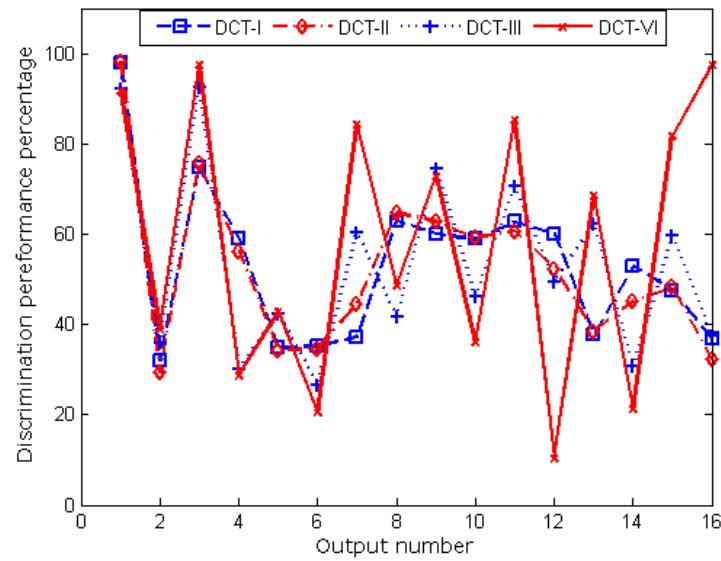

Figure 3 percentage efficiencies of the 4 DCT types

There are sixteen outputs for each type of DST or DCT, so the best output of each DST and DCT types is selected. Table 1 shows the best output of each DST type in terms of threshold values, percentage of correct discrimination for each detector and total correctness.

Table 1: the best outputs of DST types

\begin{tabular}{|c|c|c|c|c|c|}
\hline $\begin{array}{c}\text { DST- } \\
\text { type }\end{array}$ & $\begin{array}{c}\text { Out } \\
\text { put }\end{array}$ & Threshold & $\begin{array}{c}\text { LSO } \\
\text { \% }\end{array}$ & $\begin{array}{c}\text { LuYAP } \\
\text { \% }\end{array}$ & $\begin{array}{c}\text { Total } \\
\text { \% }\end{array}$ \\
\hline I & 8 & 0.053 & 93.14 & 88.3 & 90.72 \\
\hline II & 1 & 4.832 & 96.5 & 92.1 & 94.3 \\
\hline III & 16 & 0.069 & 99.34 & 98.24 & 98.79 \\
\hline IV & 1 & 3.480 & 99.46 & 96.7 & 98.08 \\
\hline
\end{tabular}

From Table 1, the best performance of DST-based techniques is the output sixteen of type III; Figure 4 shows the distribution of transformed pulses at this output.

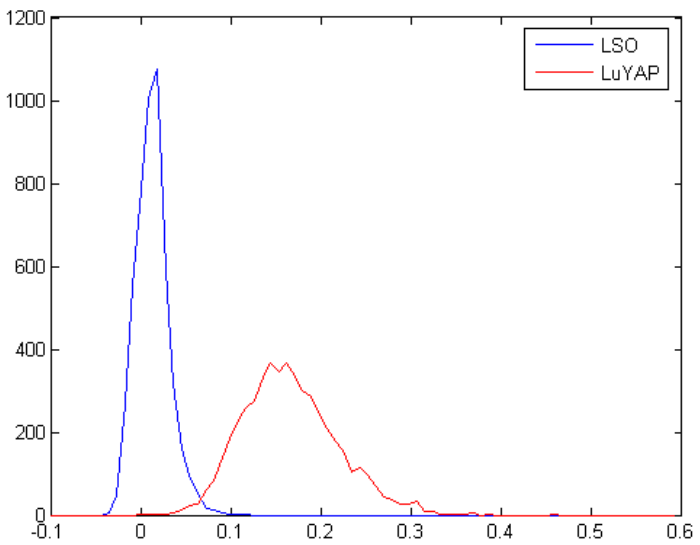

Figure 4 distribution of output 16 in DST type three

Table 2 the best outputs of DCT types

\begin{tabular}{|c|c|c|c|c|c|}
\hline $\begin{array}{c}\text { DCT- } \\
\text { type }\end{array}$ & Output & Threshold & LSO\% & $\begin{array}{c}\text { LuYAP } \\
\text { \% }\end{array}$ & Total \\
\hline I & 1 & 5.7208 & 98.38 & 97.28 & 97.83 \\
\hline II & 1 & 5.7972 & 98.54 & 97.7 & 98.12 \\
\hline III & 3 & -2.4959 & 91.92 & 93.04 & 92.48 \\
\hline IV & 3 & -2.6546 & 96.86 & 97.96 & 97.41 \\
\hline
\end{tabular}

From Table 2, the best performance of DCT-based techniques is the first output of type II. Figure 5 shows the distribution of transformed pulses at output one.

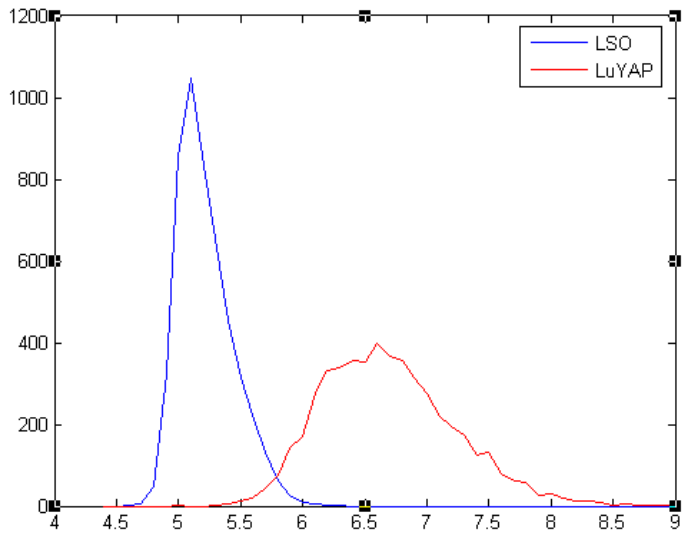

Figure 5 Histogram of the first output in DCT-II

Now, the tuned method will be studied. From Table 1 for DST, output sixteen is the best one of DST-type three, the intersection between the two distribution of LSO and LuYAP is from (0 0.1 ) as shown in Figure 4. So the threshold is chosen in this period with small step such as 0.05 then percentage of each step is calculated as shown in Table 3 
Table 3 Tuned scan of threshold values for output one of DST-IV

\begin{tabular}{|c|c|c|c|}
\hline Threshold & $\begin{array}{c}\text { LSO correct } \\
\text { \% }\end{array}$ & $\begin{array}{c}\text { LuYAP } \\
\text { correct \% }\end{array}$ & Total \% \\
\hline 0 & 26.38 & 99.98 & 63.18 \\
\hline 0.01 & 46.92 & 99.96 & 73.44 \\
\hline 0.02 & 71.36 & 99.9 & 85.63 \\
\hline 0.03 & 86.2 & 99.84 & 93.02 \\
\hline 0.04 & 93.18 & 99.74 & 96.46 \\
\hline 0.05 & 96.7 & 99.46 & 98.08 \\
\hline 0.06 & 98.56 & 98.84 & 98.7 \\
\hline 0.07 & 99.42 & 98.06 & 98.74 \\
\hline 0.08 & 99.64 & 96.52 & 98.08 \\
\hline 0.09 & 99.86 & 94.12 & 96.99 \\
\hline 0.1 & 99.92 & 90.42 & 95.17 \\
\hline
\end{tabular}

The best value in the previous table is $98.74 \%$ while the statistical method gives $98.79 \%$ however to improve the trial method the step should be very small, such as equal to .001 after that percentage is calculated for these 100 values, the best percentage at threshold of 0.066 with percentage $98.82 \%$ which slightly higher than calculated value of equation 9.

In Table 2 for DCT-types, the output one of type two which is the best correct percentage occurred, so for comparison the threshold value of this level will be studied. Table 4 shows the calculated tuned threshold values for first output of the DCT-II.

Table 4 Tuned scan of threshold values for the first output of DCT-II

\begin{tabular}{|c|c|c|c|}
\hline Threshold & $\begin{array}{c}\text { LSO correct } \\
\text { \% }\end{array}$ & $\begin{array}{c}\text { LuYAP } \\
\text { correct \% }\end{array}$ & Total \% \\
\hline 5.5 & 88.1 & 99.78 & 93.94 \\
\hline 5.55 & 90.78 & 99.6 & 95.19 \\
\hline 5.6 & 93.38 & 99.38 & 96.38 \\
\hline 5.65 & 95.22 & 99.2 & 97.21 \\
\hline 5.7 & 96.86 & 98.96 & 97.91 \\
\hline 5.75 & 97.88 & 98.34 & 98.11 \\
\hline 5.8 & 98.56 & 97.68 & 98.12 \\
\hline 5.85 & 99.1 & 96.82 & 97.96 \\
\hline 5.9 & 99.38 & 95.42 & 97.4 \\
\hline 5.95 & 99.62 & 93.96 & 96.79 \\
\hline
\end{tabular}

The best discrimination value in the previous table is $98.12 \%$, which equals to the discrimination performance using the calculated threshold value from equation 9. If the step is taken equal to 0.005 the percentage will be $98.20 \%$ at threshold equals to 5.775 .

\subsection{Tuning the values of $C_{1}$ and $C_{2}$ factors}

The empirical Equation (10) calculates $\mathrm{C}_{1}$ and $\mathrm{C}_{2}$ factors which are used in the previous results of thresholds calculations. These factors can be tuned in the period $[0,2]$ to find the optimum accuracy. The step of tuning is equal to (0.01) for scan all possible values then we compare these values with calculated values using equation (10) as shown in the next table.
Table 5 Tuned and calculated $\mathrm{C} 1$ factor for best output at each transform type

\begin{tabular}{|l|c|c|c|c|}
\hline \multirow{2}{*}{ Type } & \multicolumn{2}{|c|}{ Tuned factor } & \multicolumn{2}{c|}{$\begin{array}{c}\text { Calculated factor from } \\
\text { Eq. 10 }\end{array}$} \\
\cline { 2 - 5 } & C1 & $\begin{array}{c}\text { Total } \\
\text { percentage }\end{array}$ & C1 & $\begin{array}{c}\text { Total } \\
\text { percentage }\end{array}$ \\
\hline DST-I- 8 & 0.83 & 90.90 & 0.905 & 90.72 \\
\hline DST-II-1 & 0.89 & 94.36 & 0.820 & 94.30 \\
\hline DST-III-16 & 0.79 & 98.90 & 0.742 & 98.79 \\
\hline DSTIV-1 & 0.91 & 98.16 & 0.811 & 98.08 \\
\hline DCT-I-1 & 0.81 & 97.84 & 0.787 & 97.83 \\
\hline DCT-III-1 & 0.82 & 98.20 & 0.788 & 98.12 \\
\hline DCT-III-3 & 1.06 & 92.81 & 0.908 & 92.48 \\
\hline DCT-IV-3 & 0.82 & 97.42 & 0.832 & 97.41 \\
\hline
\end{tabular}

From the previous table, many details can be concluded, such as total efficiency percentages are approximately the same and factor $\mathrm{C} 1$ is between $[0.79,1.06]$.

In order to evaluate the proposed algorithm, results of the previous papers are studied as in [10]; their algorithm was based on wavelet and FFT. The FFT-based PSD uses parallel 16-point serial-bit input and needs complex multiplications but this algorithm results for discriminate the LSO from the LuYAP pulses is a reliability of $99.3 \%$.On the other hand, DWT -based algorithm reliability is of $97.18 \%$. The presented work shows that the DST-type 3 gives the best PSD efficiency of $98.79 \%$, while DCT-type 2 gives $98.12 \%$. These two algorithms avoid the difficulty of FFT computations and give percent nearly equal to the FFT-based algorithm.

\section{CONCLUSION}

Comparing the computing accuracy results from the statistical equation and the computing methods; the trial and error method is slightly better than the statistical equation for most the DST and DST types families, see table 1 and table 2. However, the statistical equation is recommended for fast processing.

The distribution of LSO data is sharper than distribution of LuYAP data, thus factors $C_{1}$ and $C_{2}$ in equation (9) substitute the difference in threshold calculation. The total discrimination efficiency using calculated factors $\mathrm{C}_{1}$ and $\mathrm{C}_{2}$ from empirical equations 10 and 11 are very similar to those acquired using the tuned factors $\mathrm{C}_{1}$ and $\mathrm{C}_{2}$ as shown in table 5 .

The discrimination performance range is from $98.79 \%$ to $90.72 \%$, for DST types. On the other hand, for DCT this range is from $98.12 \%$ to $92.48 \%$. The best result in DST-III occurred at the last output which corresponds to the highest frequency component in the pulse that indicates the decay of the pulse. On the other hand, the best percentage of DCT-types is in the earliest output that indicates the lowest frequency component in pulses. Finally, the comparison between DST types and DCT types gives that the output sixteen of DST-III performs $98.79 \%$ successful discrimination rate. Thus, DST-III is recommended by this investigation to be the best of the DST and DCT types for discrimination. 


\section{REFERENCES}

[1] M. Streun, G. Brandenburg, H. Larue, C. Parl, and K. Ziemons. "The Data Acquisition System of Clear PET Neuron A Small Animal PET Scanner" IEEE Trans. Nucl. Sci., vol. 53, no. 3, pp. 700-703, Jun. 2006.

[2] S. Jastaniah and P. Sellin, "Digital techniques for $n / \gamma$ pulse shape discrimination and capture-gated neutron spectroscopy using liquid scintillators". Nuclear Instruments and Methods in Physics Research A 517 (2004) pp 202-210

[3] Y. Kaschucka and B. Espositob, "Neutron/ $\gamma$-ray digital pulse shape discrimination with organic scintillators". Nuclear Instruments and Methods in Physics Research A 551 (2005) 420-428

[4] S. Yousefi and L. Lucchese, "Digital Pulse Shape Discrimination in Triple-Layer Phoswich Detectors Using Fuzzy Logic". IEEE Transactions on Nuclear Science, Vol. 55, No. 5, October 2008

[5] W. Skulski and M. Momayezi, "Particle identification in CsI:Tl using digital pulse shape analysis," Nucl. . Instrum. Methods Phys. Res. A, vol. A458, no. 3, pp. 759-771, 2001.

[6] R. Aryaeinejad, K John and F David "Comparison Between Digital and Analog Pulse Shape Discrimination Techniques For Neutron and Gamma Ray Separation", 2005 IEEE Nuclear Science Symposium Conference Record.

[7] P. Chandrikamohan and A. DeVol, "Comparison of Pulse Shape Discrimination Methods for Phoswich and CsI:Tl Detectors" 398 IEEE Transactions on Nuclear Science, Vol. 54, No. 2, April 2007

[8] H. Saleh, "Improved Techniques For Digital Signal Processing Algorithms And Their Efficient Realizations Using Field Programmable Gate Array". Ph.D Thiess, Faculty of Engineering at Cairo University" (2003).

[9] R. Fontaine et al, "Real time digital signal processing implementation for an APD-based PET scanner with Phoswich detectors" IEEE Trans. Nucl. Sci., vol. 53, no.3, pp. 784-788, Jun. 2006.

[10] Xiaowen Kang et al, “ Comparing Crystal Identification Algorithms for PET Block Detectors". 2008 IEEE Nuclear Science Symposium Conference Record

[11] R. Fontaine et al, "Digital Signal Processing Applied to Crystal Identification In Positron Emission Tomography Dedicated to Small Animals" Nuclear Instruments and Methods in Physics Research A 571 (2007) 385-388

[12] A. Arafa, H. Saleh, M. Ashour, A. Salem, "FFT- and DWT-based FPGA Realization of Pulse Shape
Discrimination in PET System". 4th IEEE International Conference on Design \&Technology of Integrated Systems in Nanoscale Era, DTIS09, 6-7 APRIL 2009.

[13] H. Semmaoui et al, "Wavelets-Based Crystal Identification of Phoswich Detectors for Small-Animal PET". IEEE Transactions on Nuclear Science, Vol. 55, No. 3, June 2008

[14] S. Yousefi and L. Lucchese, "A Wavelet-Based Pulse Shape Discrimination Method For Simultaneous Beta And Gamma Spectroscopy" Nuclear Instruments and Methods in Physics Research A 599 (2009) 66-73

[15] H. Semmaoui, R. Lecomte and R. Fontaine "A CrossCorrelation Method for Crystal Identification in APDBased Phoswich Detectors Used In Small Animal PET Scanner". Nuclear Instruments and Methods in Physics Research A 597 (2008) 238-241

[16] L. MacDonald and M. Dahlbom, "Parallax correction in PET using depth of interaction information," IEEE Trans. Nucl. Sci., vol. 45, pp. 2232-2237, 1998.

[17] K. L. Matthews, S. M. Leonard, C. E. Ordonez, D. E. Persyk, and W. Chang, "A depth-encoding anger detector using scintillating fibers," IEEE Trans. Nucl. Sci., vol. 48, pp. 1397-1402, 2001

[18] H. Murayama, H. Ishibashi, H. Uchida, T. Omura, and T.Yamashita, "Design of a depth of interaction detector with a PS-PMT for PET," IEEE Trans. Nucl. Sci., vol. 47, pp. 1045-1050, 2000.

[19] J. S. Huber, W. W. Moses, M. S. Andreaco, and O. Petterson, "An LSO scintillator array for a PET detector module with depth of interaction measurement," IEEE Trans. Nucl. Sci., vol. 48, pp. 684-688, 2001.

[20] Y. Shao and S. Cherry, "A study of depth of interaction measurement using bent optical fibers," IEEE Trans. Nucl. Sci., vol. 46, pp. 618-623, 1999.

[21] P. Jain ,B. Kumar and S. Jain ”A general design for one dimensional discrete sine transform". Analog Integrated Circ Signal Processing (2009).

[22] V. Reju et al, "Convolution Using Discrete Sine and Cosine Transforms". Signal Processing Letters, IEEE Vol 14 , Issue:7 (2007)

[23] B. Zhechev, "The Discrete Cosine Transform DCT- 4 and DCT- 8". International Conference on Computer Systems and Technologies - CompSysTech'2003

[24] Y. Yokotani and S. Oraintara "Lossless Audio Compression Using Integer Modified Discrete Cosine Transform". 2003 International Symposium on Intelligent Signal Processing and Communication Systems (ISPACS 2003). 